\title{
Stabilization of an Underactuated Surface Vessel Based on Adaptive Sliding Mode and Backstepping Control
}

\author{
Fuguang Ding, Jing Wu, and Yuanhui Wang \\ Automation College, Harbin Engineering University, Harbin 150001, China \\ Correspondence should be addressed to Yuanhui Wang; wangyuanhui2008@yahoo.com.cn
}

Received 15 November 2012; Accepted 10 February 2013

Academic Editor: Tsung-Chih Lin

Copyright (C) 2013 Fuguang Ding et al. This is an open access article distributed under the Creative Commons Attribution License, which permits unrestricted use, distribution, and reproduction in any medium, provided the original work is properly cited.

\begin{abstract}
The paper studied controlling problem of an underactuated surface vessel with unknown interferences. It proved that the control problem of underactuated surface vessel can be transformed into the stabilization analysis of two small subsystems. This controller was designed by backstepping method and adaptive sliding mode, was suitable for solving the problem of the control of higher systems, can keep the system global asymptotic stability, and can inhibit unknown interference, and boundary layer can weaken the buffeting generated by sliding mode. The unknown interference was estimated by adaptive function. Finally, the simulation results are given to demonstrate the effectiveness of the proposed control laws.
\end{abstract}

\section{Introduction}

With the rapid development of the modern shipping industry, the study about the controller of the underactuated surface vessel has received extensive attention. Since stabilization control problem of the underactuated surface vessel belongs to the field of nonlinear control, nonlinear control theory is the basic foundation of the underactuated stabilization control theory [1]. Underactuated ship system is a nonholonomic system, and the nonholonomic system which does not meet the sufficient condition of Brockett system linear approximation is not controllable; so, stabilization control of underactuated systems cannot be continuous and smooth [2]. Similarly, it cannot use output feedback or smooth state feedback on a linear system, and the linear control method is not satisfactory to the stabilization control of underactuated system.

So far, underactuated ship stabilization control problem is more studied without interferences, just like [3-7] for the case of noninterference. But for a bounded environment disturbance (or unknown disturbances) and the uncertainty of the ship systems, underactuated stabilization controller is few, except for [8], but from the research results of them shows that the antidisturbance (or uncertainty) ability of the designed controller is less than ideal. Backstepping and sliding mode control method is the combination of backstepping and sliding mode control and takes full advantages of their own characteristics in [9]. Boundedness and convergence can keep the equilibrium point; sliding mode control system can match perturbation and disturbance, and when using backstepping under less restrictive conditions, it can ensure that the closed-loop system has strong robustness. The controller exist sliding mode; so, it will cause system chattering and make control input change severely, and it cannot meet the requirements of the actual system, but boundary layer method can solve it. Adaptive function is used to cope with approximation of uncertain functions. The paper studies underactuated surface vessel, which under the conditions of bounded variable environmental interference effect. A new adaptive sliding mode and backstepping control method is designed to deal with the underactuated ship stabilization problem, and according to the experimental simulation, the designed controller is effective.

\section{Model of an Underactuated Surface Vessel}

We consider an underactuated surface vessel with simplified dynamics off-diagonal terms of the linear and nonlinear 
damping matrices which are ignored. Based on [9], the dynamics and kinematics of an underactuated surface vessel are described as follows:

$$
\begin{aligned}
& \dot{x}=u \cos (\psi)-v \sin (\psi), \\
& \dot{y}=u \sin (\psi)+v \cos (\psi), \\
& \dot{\psi}=r, \\
& \dot{u}=\frac{m_{22}}{m_{11}} v r-\frac{d_{11}}{m_{11}} u+\frac{1}{m_{11}} \tau_{1}+\frac{1}{m_{11}} \tau_{w 1}, \\
& \dot{v}=-\frac{m_{11}}{m_{22}} u r-\frac{d_{22}}{m_{22}} v+\frac{1}{m_{22}} \tau_{w 2}, \\
& \dot{r}=\frac{m_{11}-m_{22}}{m_{33}} u v-\frac{d_{33}}{m_{33}} r+\frac{1}{m_{33}} \tau_{3}+\frac{1}{m_{33}} \tau_{w 3},
\end{aligned}
$$

where $\left[\begin{array}{lll}x & y & \psi\end{array}\right]^{T}$ denote position and orientation in inertial frame, $\left[\begin{array}{lll}u & v & r\end{array}\right]^{T}$ denote linear velocities in surge sway and angular velocity in yaw, $m_{11}, m_{22}, m_{33}$ are the constants of inertia matrix, and $d_{11}, d_{22}, d_{33}$ are the constants of damping matrix, and we consider the case that the surface vessel has no side thruster; so, the control input can be described as $\left[\begin{array}{lll}\tau_{1} & 0 & \tau_{3}\end{array}\right]^{T}$, and $\left[\begin{array}{lll}\tau_{w 1} & \tau_{w 2} & \tau_{w 3}\end{array}\right]^{T}$ are the environmental disturbances in the body-fixed frame.

\section{Controller Design}

3.1. Coordinate and Feedback Transformation. In order to design the controller conveniently, the underactuated ships model will be converted to a suitable form. Based on [10], the coordinate and input transformations are as follows:

$$
\begin{aligned}
& z_{1}=x \cos \psi+y \sin \psi, \\
& z_{2}=-x \sin \psi+y \cos \psi, \\
& z_{3}=\psi \\
& z_{4}=-\frac{m_{11}}{d_{22}} u-z_{1}, \\
& z_{5}=v \\
& z_{6}=r \\
& u_{1}=\left(\frac{d_{11}}{d_{22}}-1\right) u-z_{2} z_{6}-\frac{\tau_{1}+\tau_{w 1}}{d_{22}}, \\
& u_{2}=\frac{m_{11}-m_{22}}{m_{33}} u v-\frac{d_{33}}{m_{33}} r+\frac{\tau_{3}+\tau_{w 3}}{m_{33}} .
\end{aligned}
$$

By using (2), we can transform the differential equation as follows:

$$
\begin{aligned}
& \dot{z}_{1}=-\frac{d_{22}}{m_{11}} z_{1}-\frac{d_{22}}{m_{11}} z_{4}+z_{2} z_{6}-\frac{m_{11}}{d_{22}} z_{5} z_{6}, \\
& \dot{z}_{5}=-\frac{d_{22}}{m_{22}} z_{5}+\frac{d_{22}}{m_{22}} z_{6}\left(z_{1}+z_{4}\right),
\end{aligned}
$$

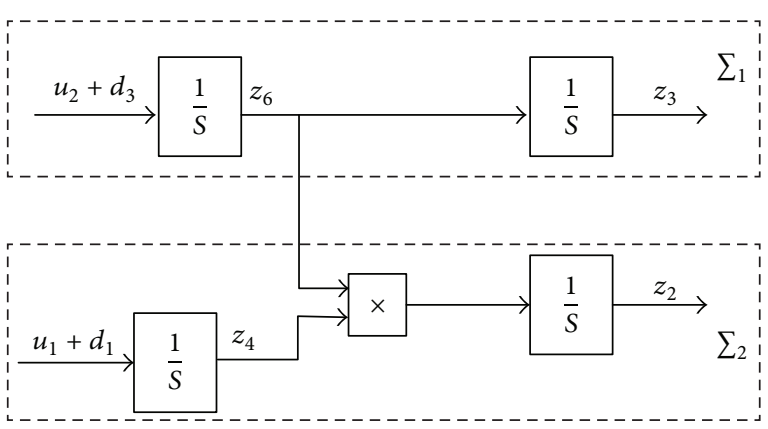

FIGURE 1: Block diagram of the system of (4).

$$
\begin{aligned}
& \dot{z}_{2}=z_{4} z_{6}+\frac{\tau_{w 2}}{d_{22}}, \\
& \dot{z}_{3}=z_{6}, \\
& \dot{z}_{4}=u_{1}+d_{1}, \\
& \dot{z}_{6}=u_{2}+d_{3},
\end{aligned}
$$

where $d_{1}=\tau_{w 1} / d_{22}$, and $d_{3}=-\tau_{w 3} / m_{33}$.

Remark 1. Because state transformation (2) is a global diffeomorphism transformation, so $\lim _{t \rightarrow \infty} z_{i}=0$ for $i=$ $1,2, \ldots, 6$ is convergent by exponential form, and thus we imply that $\lim _{t \rightarrow \infty}(\eta, V)=0$.

Proposition 2. $\lim _{t \rightarrow \infty} z_{i}=0, i=1,2, \ldots, 6$, is globally exponential stable if $\lim _{t \rightarrow \infty} z_{i}=0, i=2,3,4,6$, is globally exponential stable.

Proof. The similar proof has been given in [1].

According to Proposition 2, we know that in order to make the stabilization of underactuated surface vessels from the initial position to the origin, the system only needs to make transformation (4). It can be seen as strict feedback nonlinear subsystem, and the cascade system consists of two components which are shown in Figure 1 as follows:

$$
\begin{gathered}
\Sigma_{1}:\left\{\begin{array}{l}
\dot{z}_{3}=z_{6}, \\
\dot{z}_{6}=u_{2}+d_{3},
\end{array}\right. \\
\Sigma_{2}:\left\{\begin{array}{l}
\dot{z}_{2}=z_{4} z_{6}+\frac{\tau_{w 2}}{d_{22}}, \\
\dot{z}_{4}=u_{1}+d_{1} .
\end{array}\right.
\end{gathered}
$$

3.2. Controller Design. Figure 1 shows that the controller of underactuated surface vessels can be transformed into two single-input and single-output systems. So, we only need to design the controller to satisfy the fact that $u_{1}$ and $u_{2}$ can be controlled in three directions of the ship.

3.2.1. Design the Control Law of System $\Sigma_{1}$. Firstly, we consider the subsystem $z_{3}$ and define Lyapunov function 
$V_{1}\left(z_{3}\right)=(1 / 2) z_{3}^{2}$, and the time derivative of $V_{1}\left(z_{3}\right)$ is as follows:

$$
\dot{V}_{1}\left(z_{3}\right)=z_{3} \dot{z}_{3}=z_{3} z_{6}
$$

We take $z_{6}$ as a virtual control input of $z_{3}$ subsystem and define $z_{6}=e_{1}+\alpha_{1}\left(z_{3}\right)$. In order to ensure that the subsystem $z_{3}$ is asymptotically stable when $e_{1}=0$, we choose $\alpha_{1}\left(z_{3}\right)=-k_{1} z_{3}$, where $\alpha_{1}(0)=0$, and $k_{1}>0$. Then, we can get equations as follows:

$$
\begin{aligned}
& \dot{e}_{1}=\dot{z}_{6}-\dot{\alpha}_{1}\left(z_{3}\right)=u_{2}+d_{3}+k_{1} z_{6} \\
& \dot{V}_{1}\left(z_{3}\right)=z_{3} z_{6}=-k_{1} z_{3}^{2}+z_{3} e_{1} \leq 0 .
\end{aligned}
$$

Secondly, we consider the whole system

$$
\begin{gathered}
\dot{z}_{3}=e_{1}-k_{1} z_{3}, \\
\dot{e}_{1}=u_{2}+d_{3}+k_{1} z_{6} .
\end{gathered}
$$

We can assume the uncertain parameters $d_{3}$ exists the upper bound and choose a linear sliding mode switch function

$$
\sigma_{1}=c_{1} z_{3}+e_{1}, \quad c_{1}>0
$$

Define the Lyapunov function $V_{2}\left(z_{3}, z_{6}\right)=(1 / 2) \sigma_{1}^{2}+$ $V_{1}\left(z_{3}\right)$; the time derivative of $V_{2}\left(z_{3}, z_{6}\right)$ becomes

$$
\begin{aligned}
\dot{V}_{2}\left(z_{3}, z_{6}\right)= & -k_{1} z_{3}^{2}+z_{3} e_{1} \\
& +\sigma_{1}\left[c_{1}\left(e_{1}-k_{1} z_{3}\right)+u_{2}+d_{3}+k_{1} z_{6}\right] .
\end{aligned}
$$

Choose the control law

$$
\begin{aligned}
u_{2}= & -c_{1}\left(e_{1}-k_{1} z_{3}\right)-k_{1} z_{6} \\
& -\bar{d}_{3} \operatorname{sgn}\left(e_{1}\right)-h_{1}\left(\sigma_{1}+\beta_{1} \operatorname{sgn}\left(\sigma_{1}\right)\right),
\end{aligned}
$$

where $\operatorname{sgn}(x)$ is the sign function, $h_{1} \geq 0, \beta_{1} \geq 0$, and $\bar{d}_{3}$ is the upper bound of uncertain parameter $d_{3}$. Combining (12) into (11), we can obtain

$$
\begin{aligned}
\dot{V}_{2}\left(z_{3}, z_{6}\right) & =-k_{1} z_{3}^{2}+z_{3} e_{1}-h_{1} \sigma_{1}^{2}-h_{1} \beta_{1}\left|\sigma_{1}\right|+d_{3} \sigma_{1}-\bar{d}_{3}\left|\sigma_{1}\right| \\
& \leq-k_{1} z_{3}^{2}+z_{3} e_{1}-h_{1} \sigma_{1}^{2}-h_{1} \beta_{1}\left|\sigma_{1}\right|+\left(d_{3}-\bar{d}_{3}\right)\left|\sigma_{1}\right| \\
& \leq-k_{1} z_{3}^{2}+z_{3} e_{1}-h_{1} \sigma_{1}^{2}-h_{1} \beta_{1}\left|\sigma_{1}\right| \leq 0 .
\end{aligned}
$$

Finally, because the upper bound of the general object is difficult to predict, and in order to determine the uncertainties of the upper bound, we use the adaptive algorithm to estimate it. $\bar{d}_{3}$ is the upper bound of uncertain parameter $d_{3}$. And its adaptive rate is $\dot{\bar{d}}_{3}=\gamma_{1} \sigma_{1}$.

3.2.2. Design the Control Law of System $\Sigma_{2}$. Firstly, consider the subsystem $z_{2}$, and define Lyapunov function $V_{3}\left(z_{2}\right)=$ $(1 / 2) z_{2}^{2}$. We see $z_{4}$ as a virtual control input of subsystem $z_{2}$ and define $z_{4}=-k_{2} z_{2} \operatorname{sgn}\left(z_{6}\right)$. The time derivative of $V_{3}\left(z_{2}\right)$ is as follows:

$$
\dot{V}_{3}\left(z_{2}\right)=-k_{2} z_{2}^{2} z_{6} \operatorname{sgn}\left(z_{6}\right)=-k_{2} z_{2}^{2}\left|z_{6}\right| \leq 0, \quad k_{2}>0 .
$$

Assuming that lateral disturbance force decrease with time, and $\left|\tau_{w 2} / d_{22}\right| \leq\left|z_{2} z_{6}\right|$. In this paper, we can make $\tau_{w 2}=0$; so, $z_{2}$ is asymptotically convergent to zero when $z_{6}(0) \neq 0$ (or $z_{3}(0) \neq 0$ ). In order to make $z_{2}$ asymptotically converging to zero, we can adjust the $k_{2}$ (and $k_{1}$ ) such that the convergence rate of $z_{2}$ is greater than the convergence rate of $z_{6}\left(\right.$ or $\left.z_{3}\right)$.

Define $z_{4}=e_{2}+\alpha_{2}\left(z_{2}\right)$. In order to ensure that the subsystem $z_{2}$ is asymptotically stable when $e_{2}=0$, we choose $\alpha_{2}\left(z_{2}\right)=-k_{2} z_{2} \operatorname{sgn}\left(z_{6}\right)$, where $\alpha_{2}(0)=0$. Then, we can get equations as follows:

$$
\begin{gathered}
\dot{e}_{2}=\dot{z}_{4}-\dot{\alpha}_{2}\left(z_{2}\right)=u_{1}+d_{1}+k_{2} z_{4} z_{6} \operatorname{sgn}\left(z_{6}\right), \\
\dot{V}_{3}\left(z_{2}\right)=z_{2} z_{6}\left(e_{2}-k_{2} z_{2} \operatorname{sgn}\left(z_{6}\right)\right) \leq 0 .
\end{gathered}
$$

Secondly, consider the whole system

$$
\begin{gathered}
\dot{z}_{2}=z_{6}\left(e_{2}-k_{2} z_{2} \operatorname{sgn}\left(z_{6}\right)\right), \\
\dot{e}_{2}=u_{1}+d_{1}+k_{2} z_{4} z_{6} \operatorname{sgn}\left(z_{6}\right) .
\end{gathered}
$$

We assume the uncertain parameters $d_{1}$ exists the upper bound. Choose a linear sliding mode switch function $\sigma_{2}=$ $c_{2} z_{2}+e_{2}, c_{2}>0$. Define the Lyapunov function $V_{4}\left(z_{2}, z_{4}\right)=$ $(1 / 2) \sigma_{2}^{2}+V_{3}\left(z_{2}\right)$; the time derivative of $V_{4}\left(z_{2}, z_{4}\right)$ is as follows:

$$
\begin{aligned}
\dot{V}_{4}\left(z_{2}, z_{4}\right)= & z_{2} z_{6}\left(e_{2}-k_{2} z_{2} \operatorname{sgn}\left(z_{6}\right)\right) \\
& +\sigma_{2}\left[c_{2} z_{6}\left(e_{2}-k_{2} z_{2} \operatorname{sgn}\left(z_{6}\right)\right)\right] \\
& +\sigma_{2}\left[u_{1}+d_{1}+k_{2} z_{4} z_{6} \operatorname{sgn}\left(z_{6}\right)\right] .
\end{aligned}
$$

Choose the control law

$$
\begin{aligned}
u_{1}= & -c_{2} z_{6}\left(e_{2}-k_{2} z_{2} \operatorname{sgn}\left(z_{6}\right)\right)-k_{2} z_{4} z_{6} \operatorname{sgn}\left(z_{6}\right) \\
& -\bar{d}_{1} \operatorname{sgn}\left(e_{2}\right)-h_{2}\left(\sigma_{2}+\beta_{2} \operatorname{sgn}\left(\sigma_{2}\right)\right),
\end{aligned}
$$

where $h_{2} \geq 0, \beta_{2} \geq 0$, and $\bar{d}_{1}$ is the upper bound of uncertain parameter $d_{1}$.

Combining (18) into (17), we can obtain

$$
\begin{aligned}
\dot{V}_{4}\left(z_{2}, z_{4}\right)= & z_{2} z_{6}\left(e_{2}-k_{2} z_{2} \operatorname{sgn}\left(z_{6}\right)\right)-h_{2} \sigma_{2}^{2} \\
& \quad-h_{2} \beta_{2}\left|\sigma_{2}\right|+d_{1} \sigma_{2}-\bar{d}_{1}\left|\sigma_{2}\right| \\
\leq & z_{2} z_{6}\left(e_{2}-k_{2} z_{2} \operatorname{sgn}\left(z_{6}\right)\right)-h_{2} \sigma_{2}^{2} \\
& \quad-h_{2} \beta_{2}\left|\sigma_{2}\right|-\left(\bar{d}_{1}-d_{1}\right)\left|\sigma_{2}\right| \\
\leq & z_{2} z_{6}\left(e_{2}-k_{2} z_{2} \operatorname{sgn}\left(z_{6}\right)\right)-h_{2} \sigma_{2}^{2}-h_{2} \beta_{2}\left|\sigma_{2}\right| \leq 0 .
\end{aligned}
$$

Finally, we use the adaptive algorithm to estimate $\bar{d}_{3}$. And its adaptive rate is $\dot{\bar{d}}_{3}=\gamma_{1} \sigma_{1}$. 
3.3. Stability Analysis. Firstly, we should analyze the stability of the system $\Sigma_{1}$, defined as follows:

$$
Q_{1}=\left[\begin{array}{cc}
k_{1}+h_{1} c_{1}^{2} & h_{1} c_{1}-\frac{1}{2} \\
h_{1} c_{1}-\frac{1}{2} & h_{1}
\end{array}\right], \quad W_{1}=\left[\begin{array}{ll}
z_{3} & e_{1}
\end{array}\right]^{T}
$$

We can get

$$
\begin{gathered}
\left|Q_{1}\right|=h_{1}\left(k_{1}+h_{1} c_{1}^{2}\right)-\left(h_{1} c_{1}-\frac{1}{2}\right)^{2}=h_{1}\left(k_{1}+c_{1}\right)-\frac{1}{4} \\
W_{1}^{T} Q_{1} W_{1}=\left[\begin{array}{ll}
z_{3} & e_{1}
\end{array}\right]\left[\begin{array}{cc}
k_{1}+h_{1} c_{1}^{2} & h_{1} c_{1}-\frac{1}{2} \\
h_{1} c_{1}-\frac{1}{2} & h_{1}
\end{array}\right]\left[\begin{array}{l}
z_{3} \\
e_{1}
\end{array}\right] \\
=z_{3}^{2}\left(k_{1}+h_{1} c_{1}^{2}\right)+2 e_{1} z_{3}\left(h_{1} c_{1}-\frac{1}{2}\right)+e_{1}^{2} h_{1} \\
=k_{1} z_{3}^{2}+h_{1} c_{1}^{2} z_{3}^{2}+2 h_{1} c_{1} e_{1} z_{3}-e_{1} z_{3}+e_{1}^{2} h_{1}
\end{gathered}
$$

So, (13) can be written as follows:

$$
\dot{V}_{2}\left(z_{3}, z_{6}\right) \leq-W_{1}^{T} Q_{1} W_{1}-h_{1} \beta_{1}\left|\sigma_{1}\right| .
$$

From (21), choosing the adaptive value of $k_{1}, h_{1}$, and $c_{1}$, we can make $\left|Q_{1}\right|>0$; then, $\dot{V}_{2}\left(z_{3}, z_{6}\right) \leq 0$. The wide range of Lyapunov asymptotic stability theorem shows that the system $\Sigma_{1}$ is stable.

Secondly, system $\Sigma_{1}$ and system $\Sigma_{2}$ use the same method; so, the stability of the system $\Sigma_{2}$ can refer to system $\Sigma_{1}$.

Proposition 3. The system shown in (1) can be controlled by the input of (12) and (18), and a wide range of asymptotically stabilization can be obtained by (13) and (19).

Proof. The certification process has been given in the design steps.

From field of the theory, the motion choosing sliding mode is that parameter variations and external interference have nothing to do with the systems; so, the robustness of the system using sliding mode controller is better than the general control system. But in fact, sliding mode control can cause the system chattering for the reason of noncontinuous switch; so, the boundary layer method will be introduced to weaken this vibration. Saturation function is used to replace the ideal relay functions in the appropriate boundary layer; in other words, sat $(s)$ is used instead of sgn $(s)$ as follows:

$$
\operatorname{sat}(s)= \begin{cases}+1 & s>\Delta \\ k s & |s| \leq \Delta \\ -1 & s<-\Delta\end{cases}
$$

where $\Delta$ is the thickness of boundary layer; $k$ is a constant. And $\Delta=0.33 ; k=1$.

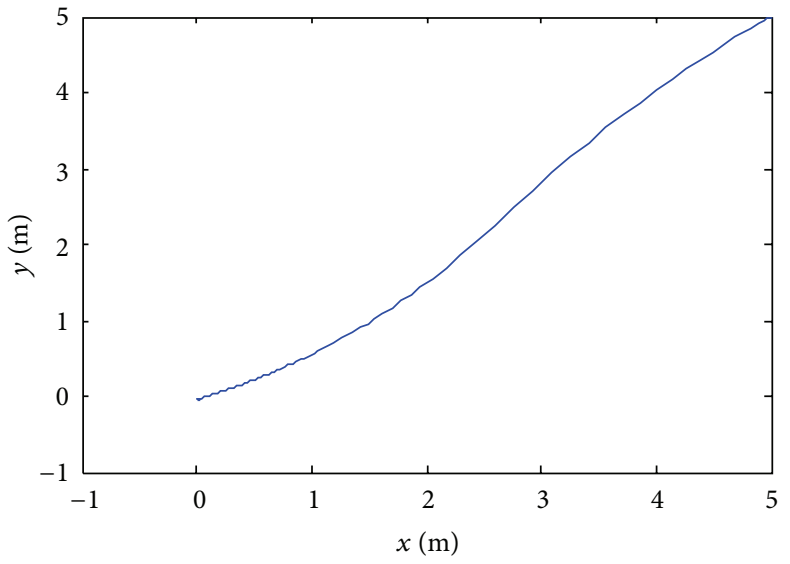

FIGURE 2: Curve of the ship's trajectory.

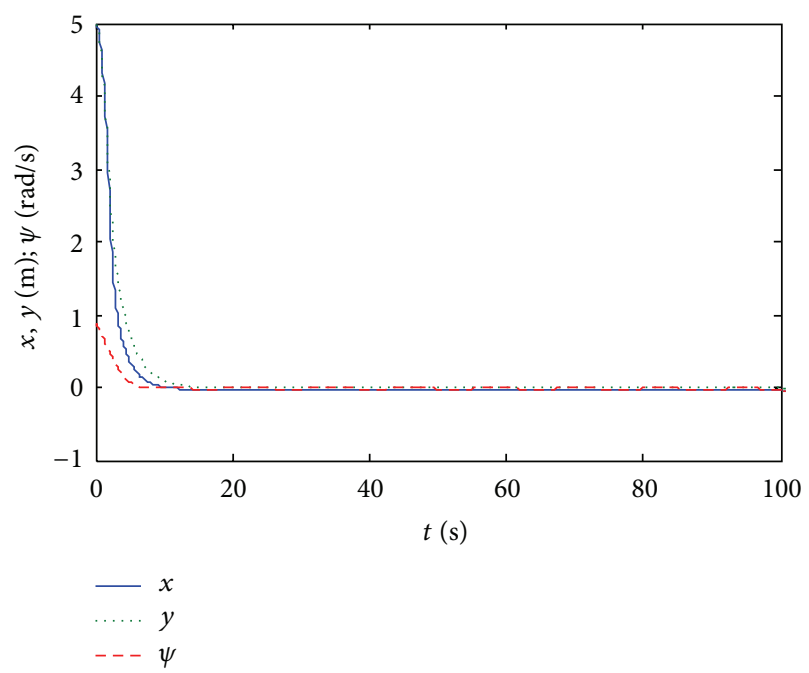

FIgURE 3: Curve of ship position and heading angle variables.

\section{Simulation}

In order to verify that the controller is able to calm from the initial point to the origin, consider an underactuated surface vessel with the model parameters in [11], and assume the initial condition to be as follows: $m_{11}=200 \mathrm{~kg}, m_{22}=250 \mathrm{~kg}$, $m_{33}=80 \mathrm{~kg} \mathrm{~m}^{2}, d_{11}=70 \mathrm{~kg} \mathrm{~s}^{-1}, d_{22}=100 \mathrm{~kg} \mathrm{~s}^{-1}, d_{33}=$ $50 \mathrm{~kg} \mathrm{~m}^{2} \mathrm{~s}^{-1}$, and $\left[x_{0}, y_{0}, \psi_{0}, u_{0}, v_{0}, r_{0}\right]^{T}=[5 \mathrm{~m}, 5 \mathrm{~m},(\pi / 4)$ $\mathrm{rad}, 0 \mathrm{~m} / \mathrm{s}, 0 \mathrm{~m} / \mathrm{s}, 0.5 \mathrm{rad} / \mathrm{s}]^{T}$.

Take the disturbances as follows: $\tau_{w 1}=50+10 \sin ((\pi /$ 6)t) $\mathrm{N}$, and $\tau_{w 2}=0 \mathrm{~N}, \tau_{w 3}=10+10 \sin ((\pi / 6) t) \mathrm{N} \cdot \mathrm{m}$.

In order to make ship at low speed operation, the longitudinal limited value of thrust is $\pm 400 \mathrm{~N}$, and torque limit value is $\pm 200 \mathrm{~N} \cdot \mathrm{m}$. The parameters of the control law are designed with the following: $k_{1}=5, c_{1}=8, h_{1}=0.2, \beta_{1}=0.1$, $k_{2}=10, c_{2}=0.1, h_{2}=6, \beta_{2}=1.8, \gamma_{1}=0.05$, and $\gamma_{2}=0.08$. Simulation results are shown as follows.

From Figure 2, we can see the trajectory of the system to be better when there exist disturbances. Designed controller can make the underactuated surface vessel stabilization. From Figures 3 and 4, although the response time is short, 


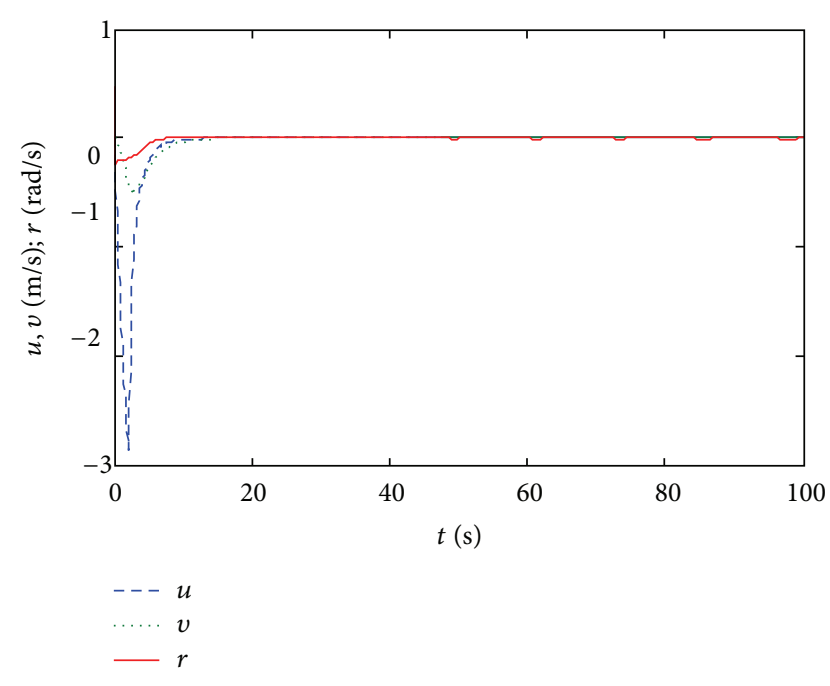

FIGURE 4: Curve of ship speed.

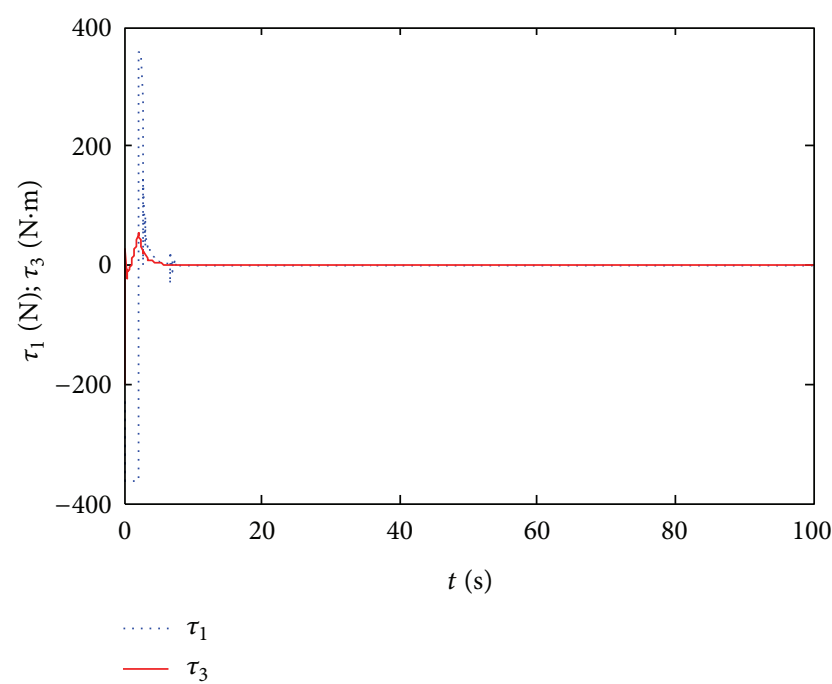

FIGURE 5: Curve of the longitudinal force and yawing moment.

designed controller can make all the states of the system stable to the origin. From Figure 5, because of introduction of the boundary layer approach to weaken the chattering phenomenon of the control force and moment, there is no change when the system is balanced, and it can save the power as well.

\section{Conclusions}

In this paper, underactuated surface vessels use the combination of adaptive sliding mode and backstepping to design the controller with the consideration of environmental disturbance and use the boundary layer method to solve the chattering problem. From the analysis of stabilization, the system is state global stabilization to the origin. The designed controller overcomes the problems of anti-to perturbation poor difficulties of the other controllers [8]; so, the designed controller improves the robustness of the system.

\section{Acknowledgments}

This work is supported by the National Natural Science Foundation of China (51209056) and the Fundamental Research Funds for the Central Universities (HEUCF 100420 and HEUCF 110430).

\section{References}

[1] K. D. Do and J. Pan, Control of Ships and Underwater Vehicles: Design for Underactuated and Nonlinear Matine Systems, Springer, London, UK, lst edition, 2009.

[2] W. Dong and Y. Guo, "Global time-varying stabilization of underactuated surface vessel," IEEE Transactions on Automatic Control, vol. 50, no. 6, pp. 859-864, 2005.

[3] Y. Liu, C. Guo, and R. Zhou, "Robust feedback stabilization control of an underactuated surface vessel," in Proceedings of WRI World Congress on Computer Science and Information Engineering (CSIE '09), pp. 46-50, March-April 2009.

[4] L. C. McNinch, H. Ashrafiuon, and K. R. Muske, "Sliding mode setpoint control of an underactuated surface vessel: simulation and experiment," in Proceedings of American Control Conference (ACC '10), pp. 5212-5217, July 2010.

[5] J. Cheng, J. Yi, and D. Zhao, "Stabilization of an underactuated surface vessel via discontinuous control," in Proceedings of the American Control Conference (ACC '07), pp. 206-211, New York, NY, USA, July 2007.

[6] Y. Yan, S. Yu, J. Du, and K. Zheng, "Observer based finitetime switched controller of an underactuated surface vessel," in Proceedings of the 3rd International Symposium on Systems and Control in Aeronautics and Astronautics (ISSCAA '10), pp. 11211125, June 2010.

[7] J. Ghommam, F. Mnif, A. Benali, and N. Derbel, "Asymptotic backstepping stabilization of an underactuated surface vessel," IEEE Transactions on Control Systems Technology, vol. 14, no. 6, pp. 1150-1157, 2006.

[8] Y. Liu, C. Guo, and R. Zhou, "Asymptotic stabilization control of an underactuated surface vessel with optimization based on genetic algorithm," in Proceedings of the 2nd International Symposium on Intelligent Information Technology Application (IITA '08), pp. 622-626, December 2008.

[9] N. Djeghali, M. Ghanes, and S. Djennoune, "Bachstepping fault tolerant control based on second order sliding mode observer: application to induction motors," in Proceedings of Decision and Control and European Control Conference, pp. 4598-4603, 2011.

[10] B.-l. Ma, "Global $\kappa$-exponential asymptotic stabilization of underactuated surface vessels," Systems \& Control Letters, vol. 58, no. 3, pp. 194-201, 2009.

[11] C. Guo, Y. Liu, and S. Zhang, "Global sliding mode based trajectory tracking control for underactuated surface vessels with uncertain dynamics," in Proceedings of the 31st Chinese Control Conference, pp. 4456-4461, 2012. 


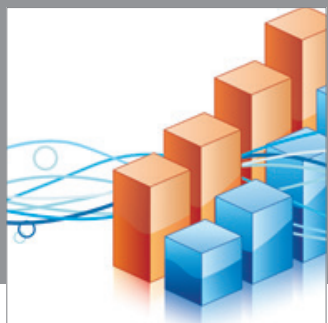

Advances in

Operations Research

mansans

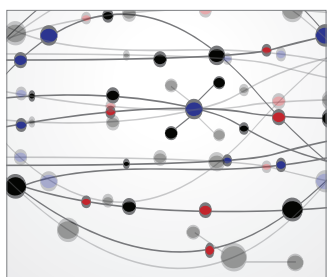

The Scientific World Journal
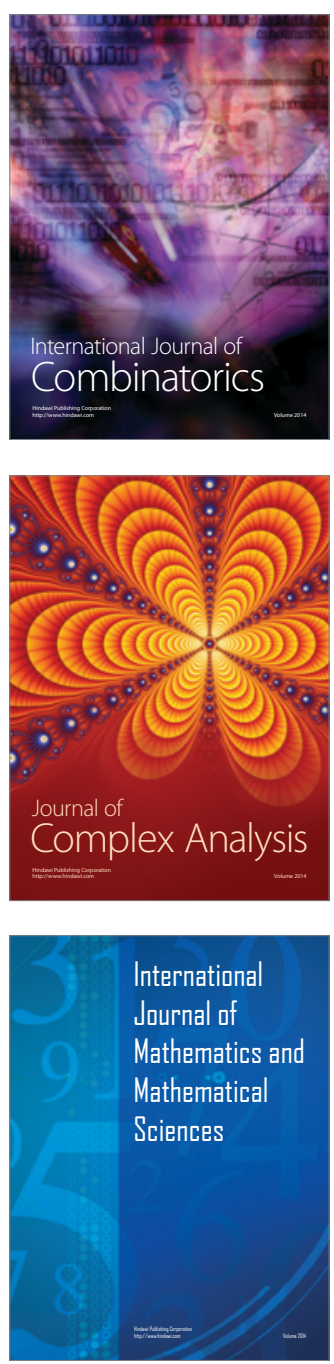
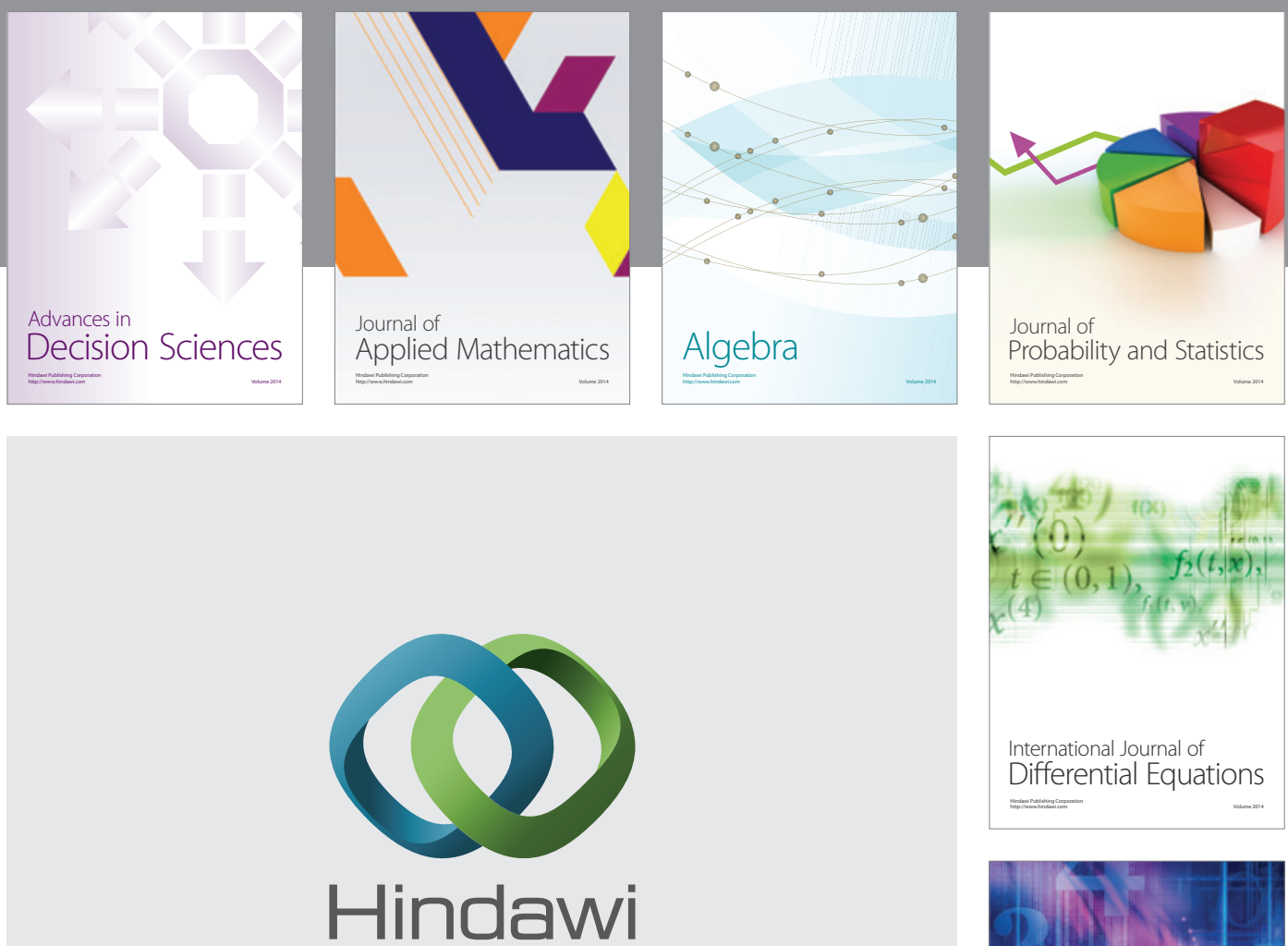

Submit your manuscripts at http://www.hindawi.com
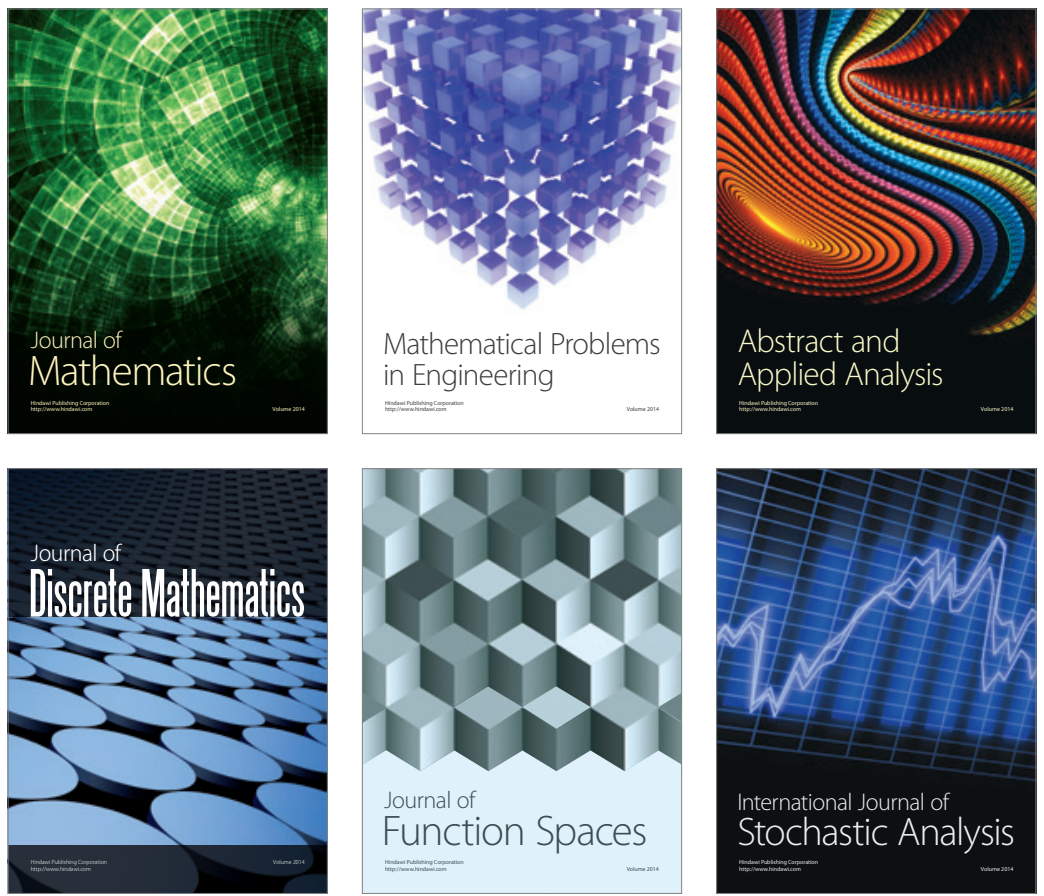

Journal of

Function Spaces

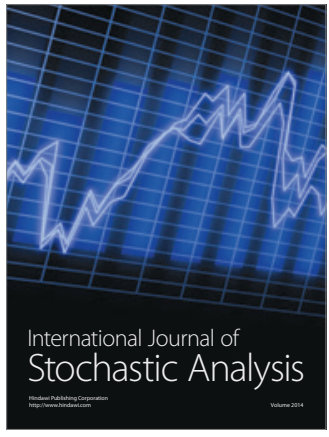

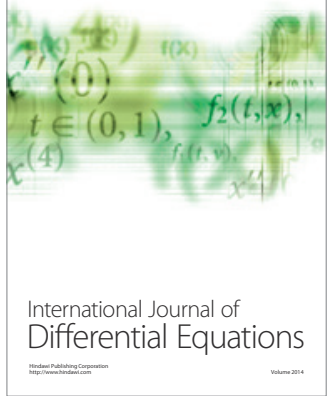
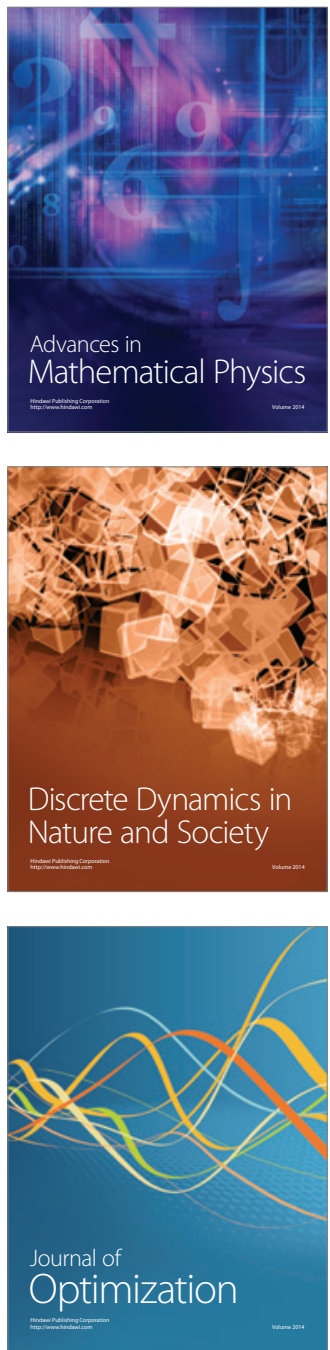